\title{
Radial endobronchial ultrasound-guided transbronchial biopsy for peripheral pulmonary malignancy: biopsy- or brushing-first?
}

\author{
Chun-Ta Huang ${ }^{1,2}$, Yi-Ju Tsai ${ }^{3}$, Chao-Chi Ho ${ }^{1 *}$ (D) and Chong-Jen Yu ${ }^{1}$
}

\begin{abstract}
Background: Radial endobronchial ultrasound (R-EBUS)-guided transbronchial biopsy (TBB) is a common diagnostic modality for peripheral pulmonary lesions; however, there is uncertainty about the optimal sequence of TBB and bronchial brushing during the procedure. Thus, we aimed to investigate whether a biopsy-first or brushing-first strategy confers a better diagnostic yield and safety signal for R-EBUS-guided procedures for peripheral pulmonary malignancy.

Methods: From January 2017 to June 2018, consecutive patients referred for R-EBUS-guided TBB and bronchial brushing of peripheral pulmonary lesions and with a final malignant diagnosis were included. Patients were placed in a biopsy-first (biopsy followed by brushing) or a brushing-first (brushing followed by biopsy) group. The outcomes of interest were the diagnostic yield and complication profile of the procedures. Multivariate logistic regression and subgroup analysis were used to assess the impact of the procedure strategy.

Results: A total of 438 patients were included and the diagnostic yield of R-EBUS-guided TBB plus brushing for peripheral pulmonary malignancy was $73 \%$. The diagnostic yield was associated with the solid lesion appearance (odds ratio [OR] 2.01; 95\% confidence interval [CI] 1.08-3.75) and R-EBUS probe position within the lesion (OR 1.92; 95\% Cl 1.08-3.42), and the yield rates were comparable between the biopsy-first and brushing-first strategies. Moreover, the safety signal did not differ between the two groups.

Conclusions: The two procedure strategies were indistinguishable in terms of diagnostic efficacy and adverse events for patients with peripheral pulmonary malignancy. Current evidence indicates that in patients with peripheral pulmonary lesions suspected of being malignant, either biopsy-first or brushing-first is a viable and acceptable diagnostic strategy during R-EBUS-guided procedures.
\end{abstract}

Keywords: Biopsy, Brushing, Diagnosis, Endobronchial ultrasound, Peripheral pulmonary lesion

\section{Background}

Diagnosis of peripheral pulmonary malignancy can be attained via a variety of modalities, such as bronchoscopy, computed tomography (CT)-guided transthoracic needle biopsy and surgery. In the past two decades, advances in bronchoscopic procedures, such as radial endobronchial ultrasound (R-EBUS), virtual bronchoscopic navigation

\footnotetext{
* Correspondence: ccho1203@ntu.edu.tw

${ }^{1}$ Department of Internal Medicine, National Taiwan University Hospital, No. 7 Chung-Shan South Rd, Taipei 100, Taiwan

Full list of author information is available at the end of the article
}

and electromagnetic navigation have made transbronchial biopsy (TBB) a more appealing and favorable approach $[1,2]$. Of the above-mentioned techniques, R-EBUSguided TBB provides a fair diagnostic yield and an excellent safety signal in diagnosing peripheral pulmonary malignancy [3-5]. In addition to $\mathrm{TBB}$, auxiliary diagnostic tools, such as bronchial brushing and washing, are commonly used to reach the cytologic diagnosis, and combining these procedures may achieve a higher diagnostic yield than TBB alone [6, 7].

C The Author(s). 2019 Open Access This article is distributed under the terms of the Creative Commons Attribution 4.0 International License (http://creativecommons.org/licenses/by/4.0/), which permits unrestricted use, distribution, and reproduction in any medium, provided you give appropriate credit to the original author(s) and the source, provide a link to the Creative Commons license, and indicate if changes were made. The Creative Commons Public Domain Dedication waiver (http://creativecommons.org/publicdomain/zero/1.0/) applies to the data made available in this article, unless otherwise stated. 
Besides the diagnostic modalities and tools, the details of the bronchoscopic procedures may affect their diagnostic yield. For instance, in bronchoscopically visible lesions, a brushing-first strategy provided a significantly higher diagnostic yield for lung cancer than a biopsyfirst strategy [8]. In a similar clinical context, bronchial washing performed before or after endobronchial biopsy did not affect the diagnostic yield of biopsy and washing [9]. Regarding R-EBUS-guided TBB of peripheral pulmonary malignancy, both biopsy- and brushing-first strategies may be applied in clinical practice $[10,11]$. However, to the best of our knowledge, no studies have been conducted to assess the ideal sequence of TBB and bronchial brushing during R-EBUS-guided procedures.

Therefore, the aims of this study were to investigate whether a biopsy-first or brushing-first strategy confers a better diagnostic yield and safety signal for R-EBUSguided TBB of peripheral pulmonary malignancy.

\section{Methods}

\section{Study setting and population}

This study was conducted at National Taiwan University Hospital, a tertiary-care referral center in Taiwan. Consecutive adult patients who underwent R-EBUS-guided TBB of peripheral pulmonary lesions from January 2017 to June 2018 were screened for eligibility. A peripheral pulmonary lesion was defined as a lesion circumscribed by lung parenchyma and invisible through conventional bronchoscopy [12]. Criteria for inclusion in this study were (a) lesions with a final diagnosis of malignancy, either primary or metastatic and (b) both TBB and bronchial brushing performed during a single bronchoscopic session. The Research Ethics Committee of National Taiwan University Hospital approved the protocol and waived informed consent given the retrospective nature of the study and the lack of patient safety concerns.

\section{Bronchoscopic procedures}

The bronchoscopic exam was primarily conducted by pulmonary fellows, as previously described, under the supervision of eight rotating pulmonary faculty in attendance [13, 14]. In brief, conventional bronchoscopy (BF-1 T260; Olympus Medical Systems Corp., Tokyo, Japan) was first performed to inspect the tracheobronchial tree after the patient received local anesthesia with lidocaine in the upper airway and intramuscular fentanyl for analgesia. Then, a $20-\mathrm{MHz}$ radial-type ultrasonic probe (UM-S20-20R; Olympus Medical Systems Corp.), equipped with an endoscopic ultrasound center (EUM30S; Olympus Medical Systems Corp.), was used to locate the peripheral pulmonary lesion, and R-EBUSguided TBB and bronchial brushing were performed.

The biopsy was taken with a cup forceps (Micro-Tech Co. Ltd., Jiangsu, China), and was repeated until adequate tissue samples were collected. Bronchial brushing was performed with a 2-mm brush (ConMed Corp., New York, United States) and a few back-and-forth movements were used to retrieve the samples. The biopsy specimens were put in $10 \%$ formalin and transported to the histopathology laboratory for analysis. Tissue samples obtained by bronchial brushing were smeared onto glass slides, air-dried at room temperature and sent for cytology exam. During the study period, fluoroscopic guidance and rapid on-site evaluation (ROSE) were not utilized at our institution, and biopsy and brushing specimens were read independently by the pathologist and cytopathologist, respectively. A biopsy-first strategy (biopsy followed by brushing) or a brushing-first strategy (brushing followed by biopsy) was chosen by the in-charge faculty based on the day of the week on which the procedure was conducted. On Monday and Thursday, it would be a biopsy-first strategy, and on Tuesday and Wednesday, a brushing-first strategy.

\section{Data collection and follow-up}

The primary outcome was the diagnostic yield of REBUS-guided TBB plus brushing for peripheral pulmonary malignancy; another outcome of interest was the incidence of procedure-related complications. Patient records and images were reviewed to obtain the following information: demographics, lesion size, location and appearance, absence or presence of a CT bronchus sign, probe position, procedure-related complications, and histopathologic and cytologic diagnosis. Lesion size was measured as the largest diameter on axial CT films. Lesion location was divided into five anatomic lobes. Lesion appearance was categorized as solid or non-solid (partly solid, pure ground-glass and cavitary). A CT bronchus sign was present if one or more bronchi leading directly to the peripheral pulmonary malignancy were identified on CT [15]. Probe position was classified as within, adjacent to or outside the peripheral pulmonary malignancy, as described previously [11]. Procedure-related complications included bleeding, pneumothorax, hemodynamic instability and bronchospasm. Self-limited bleeding was not counted as a complication in this study. Following R-EBUS-guided procedures, non-diagnostic lesions were subjected to CT-guided transthoracic needle biopsy, surgery, biopsy of other sites or repeat R-EBUS-guided TBB to pursue a definitive diagnosis of peripheral pulmonary malignancy.

\section{Statistical analysis}

Between-group comparisons were performed using $\chi^{2}$ or Fisher's exact test for categorical variables and independent samples t-test for numerical variables. A multivariate logistic regression model was constructed with the diagnostic yield of TBB plus brushing as the outcome 
variable predicted by the procedure strategy (biopsy-first vs. brushing-first), using all relevant covariates without model selection. To explore possible effect modification by lesion appearance, size and location, and probe position based on biologic plausibility, we used stratified analysis to estimate the diagnostic odds in each subgroup. $P$ values $<0.05$ were considered statistically significant and all tests were two-sided. All statistical analyses were performed using SPSS (version 20.0, IBM Corp.; Armonk, NY, US) or Stata (version 11, StataCorp.; TX, US).

\section{Results}

\section{Study subjects}

During the study period, a total of 438 patients with peripheral pulmonary malignancy were included for analysis. The average age of the study population was $66 \pm 12$ years, and slightly more than half of the subjects were male $(N=239,55 \%)$. The mean diameter of the peripheral pulmonary malignancy was $37 \pm 16 \mathrm{~mm}$. An approximately equal number of malignant lesions were distributed between the upper lobes $(N=236,54 \%)$ and the middle/lower lobes $(N=202,46 \%)$, and the majority $(N=389,89 \%)$ of them appeared solid on CT scans. Under most circumstances, the R-EBUS probe can be positioned within the malignant lesions $(N=375,86 \%)$. The leading pathologic diagnoses in our study cohort were lung adenocarcinoma $(N=312,71 \%)$, non-small cell lung cancer $(N=49,11 \%)$ and lung squamous cell carcinoma $(N=36,8.2 \%)$.

Table 1 shows the comparisons of clinical features between patients in the biopsy-first and brushing-first groups. The only characteristic that differed between the two groups was the probe location, i.e., the R-EBUS probe was more likely to be placed within the malignancy in the brushing-first group than in the biopsy-first group $(90 \%$ vs. $81 \%, P=0.004)$.

\section{Diagnostic yield of TBB plus brushing}

The overall diagnostic yield of TBB plus brushing for peripheral pulmonary malignancy was $73 \%$. The diagnostic yield was associated with the CT appearance of the malignancy and probe location (Table 2). The diagnostic yield was not affected by the procedure strategy, lobar location, lesion size or pathology of the malignancy. In the multivariate analysis (Table 3), patients with solid peripheral pulmonary malignancy (odds ratio [OR] 2.01; 95\% confidence interval [CI] 1.08-3.75) and an R-EBUS probe positioned within the lesion (OR 1.92; 95\% CI 1.08-3.42) were more likely to have the diagnosis achieved by R-EBUS-guided procedures. Figure 1 shows the results of pre-specified subgroup analyses. The diagnostic yield of the R-EBUS-guided procedures did not vary significantly between the biopsy-first and brushing-first groups across all subgroups.

\section{Safety}

No procedure-related mortality was observed in this study. Overall, complications occurred in $30(6.8 \%)$ of the 438 patients (Table 4 ). The most commonly encountered complication was bleeding $(N=21,4.8 \%)$, which was treated with instillation of topical epinephrine $(N=17)$ or bronchoscope wedge $(N=4)$. Other complications included pneumothorax $(N=6)$, unstable hemodynamics $(N=2)$ and bronchospasm $(N=1)$. Two R-EBUS-guided procedures were prematurely terminated due to the development of complications. The incidence of overall or individual complications was similar in both groups.

\section{Discussion}

The present work is the first study to compare the diagnostic yield and complications of R-EBUS-guided TBB plus brushing for peripheral pulmonary malignancy using a biopsy-first or brushing-first strategy. The main findings of our study are as follows: (a) the overall diagnostic yield of TBB plus brushing of peripheral pulmonary malignancy was 73\%; (b) a biopsy-first strategy provided diagnostic sensitivity for malignancy similar to a brushing-first strategy; (c) solid lesion appearance and position of the R-EBUS probe within the lesion were two features significantly associated with the diagnostic yield of TBB plus brushing; (d) the overall complication rate was $6.8 \%$, with hemorrhage being the most common complication; (e) the safety signal did not differ between the biopsy-first and brushing-first groups.

The most important finding in this study is that the two strategies resulted in a comparable diagnostic yield for R-EBUS-guided procedures for peripheral pulmonary malignancy. In fact, most of the previous reports regarding TBB and bronchial brushing with the assistance of R-EBUS did not specify the sequence of the procedures [6, 16-18]. Kurimoto et al. adopted a brushing-first strategy for TBB and reported a high detection rate of $81 \%$ for peripheral pulmonary malignancy [11]; on the other hand, Roth et al. performed TBB prior to bronchial brushing and the diagnostic yield for malignancy was less than $50 \%$ [7]. A recent study by Hou et al. examined the optimal sequence of forceps biopsy and bronchial brushing of visible endobronchial lung cancer and found a significantly higher diagnostic rate $(87 \%)$ in the brushing-first group compared to the biopsy-first group (79\%) [8]. The authors rationalized their results by suggesting that endobronchial tumors can be more difficult to identify and sample after biopsy, since hemorrhage may contaminate the field available for brushing [8]. In contrast to previous experience with bronchoscopically visible lesions, our study showed a 
Table 1 Clinical characteristics and final diagnosis of the study population

\begin{tabular}{|c|c|c|c|}
\hline \multirow[t]{2}{*}{ Characteristics } & Biopsy-first group & Brushing-first group & \multirow{2}{*}{$\begin{array}{l}P \\
\text { value }\end{array}$} \\
\hline & $N=219$ & $N=219$ & \\
\hline Age, years & $66 \pm 13$ & $65 \pm 11$ & 0.344 \\
\hline$\geq 65$ & $123(56)$ & $119(54)$ & 0.701 \\
\hline \multicolumn{4}{|l|}{ Gender } \\
\hline Male & $120(55)$ & $119(54)$ & 0.924 \\
\hline \multicolumn{4}{|l|}{ Lobar location } \\
\hline Right upper lobe & $63(29)$ & $52(24)$ & \multirow[t]{5}{*}{0.547} \\
\hline Right middle lobe & $18(8.2)$ & $13(5.9)$ & \\
\hline Right lower lobe & $42(19)$ & $49(22)$ & \\
\hline Left upper lobe & $56(26)$ & $65(30)$ & \\
\hline Left lower lobe & $40(18)$ & $40(18)$ & \\
\hline Lesion size, mm & $36 \pm 16$ & $37 \pm 17$ & 0.734 \\
\hline$\leq 20$ & $22(10)$ & $29(13)$ & 0.297 \\
\hline$>20$ & $197(90)$ & $190(87)$ & \\
\hline \multicolumn{4}{|l|}{ CT appearance } \\
\hline Solid & $197(90)$ & $192(88)$ & \multirow[t]{2}{*}{0.448} \\
\hline Non-solid & $22(10)$ & $27(12)$ & \\
\hline \multicolumn{4}{|l|}{$\mathrm{CT}$ bronchus sign } \\
\hline Presence & $215(98)$ & $216(99)$ & \multirow[t]{2}{*}{0.999} \\
\hline Absence & $4(1.8)$ & $3(1.4)$ & \\
\hline \multicolumn{4}{|l|}{ Probe location } \\
\hline Within & $177(81)$ & $198(90)$ & \multirow[t]{2}{*}{0.004} \\
\hline Adjacent to or outside & $42(19)$ & $21(9.6)$ & \\
\hline \multicolumn{4}{|l|}{ Lesion pathology } \\
\hline Adenocarcinoma & $162(74)$ & $150(69)$ & \multirow[t]{6}{*}{0.656} \\
\hline Squamous cell carcinoma & $18(8.2)$ & $18(8.2)$ & \\
\hline Small cell carcinoma & $7(3.2)$ & $8(3.7)$ & \\
\hline Non-small cell carcinoma & $22(10)$ & $27(12)$ & \\
\hline Metastasis & $5(2.3)$ & $11(5.0)$ & \\
\hline Others & $5(2.3)$ & $5(2.3)$ & \\
\hline Diagnostic yield & $158(72)$ & $162(74)$ & 0.667 \\
\hline
\end{tabular}

CT Computed tomography

similar diagnostic yield for peripheral pulmonary malignancy in both the biopsy-first and brushing-first groups. One speculation is that with the guidance of $\mathrm{R}$ EBUS, bronchial brushing of peripheral pulmonary lesions can be performed without visual aids, and postbiopsy bleeding will not obscure their visualization; thus, the procedure may be performed either before or after TBB. The other is that in experienced hands [4], a high quality R-EBUS-guided procedure could be performed to achieve a superior diagnostic sensitivity to peripheral pulmonary malignancy in both biopsy-first and brushing-first groups.

Our diagnostic sensitivity for peripheral pulmonary malignancy using TBB was consistent with that of previous studies of R-EBUS for malignant lesions, in which the diagnostic yield of R-EBUS ranged from 47 to $81 \%$ [6, 11, $16,19-22]$. The prevalence of malignancy present in the population being studied is a well-recognized explanation for the observed differences in the diagnostic sensitivity of R-EBUS-guided TBB for peripheral pulmonary lesions [5]. However, a wide variation in the yield of TBB still exists, even though only a subset of malignant lesions are chosen for evaluation in the literature. This heterogeneity may be explained by the discrepancies in other characteristics related to the cases, such as lesion size, personnel and institutional experience, and concomitant use of additional tools, like ROSE. The lowest diagnostic yield of $47 \%$ was observed in a study that included only solitary pulmonary 
Table 2 Variables associated with the diagnostic yield of radial endobronchial ultrasound-guided transbronchial procedures

\begin{tabular}{|c|c|c|c|}
\hline \multirow[t]{2}{*}{ Characteristics } & \multicolumn{2}{|c|}{ Diagnosed by R-EBUS-guided transbronchial procedures } & \multirow{2}{*}{$\begin{array}{l}P \\
\text { value }\end{array}$} \\
\hline & No $(N=118)$ & Yes $(N=320)$ & \\
\hline \multicolumn{4}{|l|}{ Procedure strategy } \\
\hline Biopsy-first & $61(28)$ & $158(72)$ & \multirow[t]{2}{*}{0.667} \\
\hline Brushing-first & $57(26)$ & $162(72)$ & \\
\hline \multicolumn{4}{|l|}{ Age, years } \\
\hline$<65$ & $50(42)$ & $146(46)$ & \multirow[t]{2}{*}{0.544} \\
\hline$\geq 65$ & $68(58)$ & $174(54)$ & \\
\hline \multicolumn{4}{|l|}{ Gender } \\
\hline Male & $56(48)$ & $183(57)$ & \multirow[t]{2}{*}{0.070} \\
\hline Female & $62(53)$ & $137(43)$ & \\
\hline \multicolumn{4}{|l|}{ Lobar location } \\
\hline Upper lobes & $56(48)$ & $146(46)$ & \multirow[t]{2}{*}{0.733} \\
\hline Non-upper lobes & $62(53)$ & $174(54)$ & \\
\hline \multicolumn{4}{|l|}{ Lesion size, $\mathrm{mm}$} \\
\hline$\leq 20$ & $16(14)$ & $35(11)$ & \multirow[t]{2}{*}{0.448} \\
\hline$>20$ & $102(86)$ & $285(89)$ & \\
\hline \multicolumn{4}{|l|}{ CT appearance } \\
\hline Solid & $98(83)$ & $291(91)$ & \multirow[t]{2}{*}{0.020} \\
\hline Non-solid & $20(17)$ & $29(9.1)$ & \\
\hline \multicolumn{4}{|l|}{ Probe location } \\
\hline Within & $93(79)$ & $282(88)$ & \multirow[t]{2}{*}{0.014} \\
\hline Adjacent to or outside & $25(21)$ & $38(12)$ & \\
\hline \multicolumn{4}{|l|}{ Lesion pathology } \\
\hline Adenocarcinoma & $85(72)$ & $227(71)$ & \multirow[t]{6}{*}{0.253} \\
\hline Non-small cell carcinoma & $8(6.8)$ & $41(13)$ & \\
\hline Squamous cell carcinoma & $11(9.3)$ & $5(7.8)$ & \\
\hline Small cell carcinoma & $3(2.5)$ & $12(3.8)$ & \\
\hline Metastasis & $7(5.9)$ & $9(2.8)$ & \\
\hline Others & $4(3.4)$ & $6(1.9)$ & \\
\hline
\end{tabular}

CT Computed tomography, R-EBUS Radial endobronchial ultrasound

Table 3 Multivariate logistic analysis of clinical features associated with the diagnostic yield of radial endobronchial ultrasoundguided transbronchial procedures

\begin{tabular}{|c|c|c|c|c|}
\hline Variables & & OR & $95 \% \mathrm{Cl}$ & $P$ value \\
\hline Procedure strategy & Brushing-first vs. Biopsy-first & 1.05 & $0.68-1.62$ & 0.836 \\
\hline Appearance & Solid vs. Non-solid & 2.01 & $1.08-3.75$ & 0.029 \\
\hline Probe position & Within vs. Adjacent to or outside & 1.92 & $1.08-3.42$ & 0.026 \\
\hline Lesion size & $>20 \mathrm{~mm}$ vs. $\leq 20 \mathrm{~mm}$ & 0.92 & $0.60-1.42$ & 0.715 \\
\hline Lesion location & Non-upper lobes vs. Upper lobes & 1.05 & $0.54-2.03$ & 0.887 \\
\hline
\end{tabular}




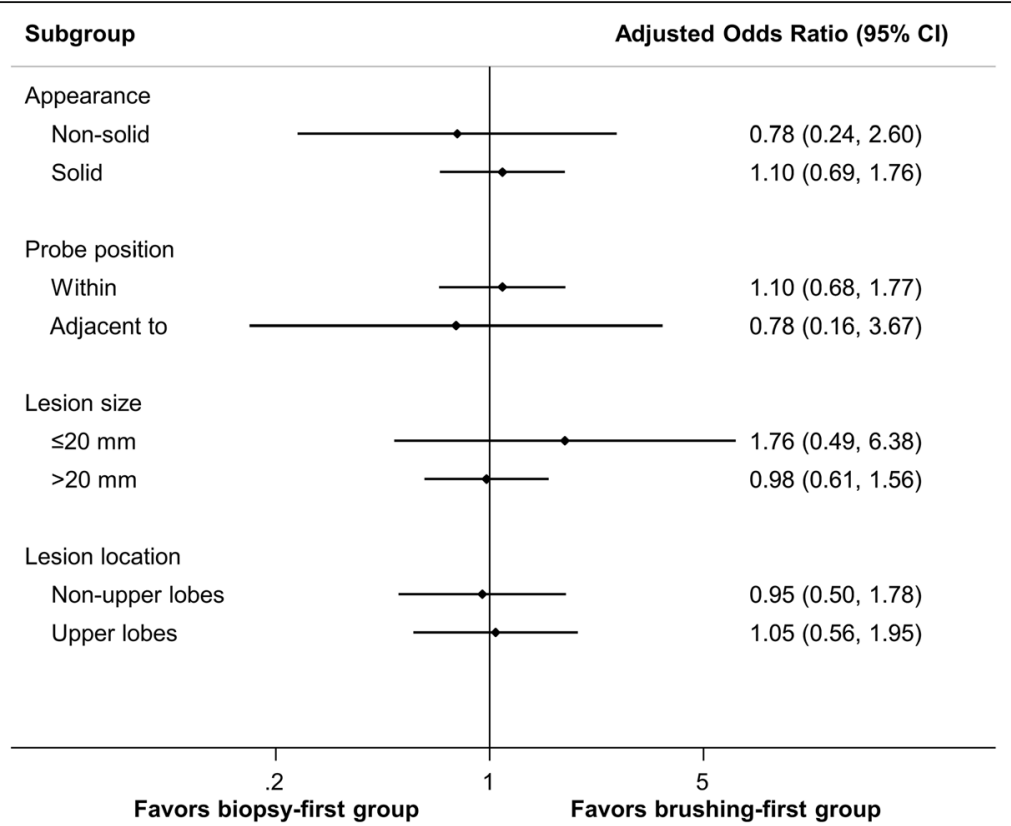

Fig. 1 Subgroup analysis of the diagnostic yield of radial endobronchial ultrasound-guided transbronchial procedures in the biopsy-first group compared to the brushing-first groupCl, confidence interval.

nodules smaller than $20 \mathrm{~mm}$ [22], and undoubtedly, procedural experience improves the performance of R-EBUSguided TBB [4]. Moreover, the application of ROSE has been shown to improve the diagnostic yield of TBB under R-EBUS guidance [23]. Therefore, it is important to consider patient and staff factors, and auxiliary modalities used when assessing how a certain technology like REBUS performs in clinical practice.

In a recent study by Chen, et al. [24], the diagnostic yield of R-EBUS-guided procedures for peripheral pulmonary lesions was positively correlated with the lesion size. Also, a meta-analysis found that lesion size was a significant determining feature in TBB performance [25]. However, the present study did not show such a finding. Our study population was composed solely of those with malignant pathology, and the procedures were conducted by a well- developed and experienced team. These specific settings may partly explain the discrepancy between our findings and others.

Safety is certainly a concern when choosing a modality or procedure. Consistent with prior studies [3,26], a favorable safety profile with no mortality or sequelae with the use of R-EBUS-guided TBB for peripheral pulmonary malignancy was observed in this work. Pneumothorax is a well-known and potentially catastrophic complication after TBB, and our occurrence rate of $1.4 \%$ lies on the low end of those previously reported: 0 to $5.1 \%[3,6,10,11,17,20-22,26]$. Bleeding is another major complication of TBB; however, its incidence is more difficult to compare across studies given the wide variation in definitions. On average, an incidence rate of $0.7 \%$ was reported for procedure-

Table 4 Complications and their management during radial endobronchial ultrasound-guided transbronchial procedures

\begin{tabular}{|c|c|c|c|}
\hline \multirow[t]{2}{*}{ Events } & Biopsy-first group & Brushing-first group & \multirow{2}{*}{$\begin{array}{l}P \\
\text { value }\end{array}$} \\
\hline & $N=219$ & $N=219$ & \\
\hline Overall & $16(7.3)$ & $14(6.4)$ & 0.705 \\
\hline Bleeding & $12(5.5)$ & $9(4.1)$ & 0.502 \\
\hline Topical epinephrine & $10(4.6)$ & $7(3.2)$ & 0.458 \\
\hline Wedging bronchoscope & $2(0.9)$ & $2(0.9)$ & 0.999 \\
\hline Pneumothorax & $3(1.4)$ & $3(1.4)$ & 0.999 \\
\hline Hemodynamic instability & $1(0.5)$ & $1(0.5)$ & 0.999 \\
\hline Bronchospasm & $0(0)$ & $1(0.5)$ & 0.999 \\
\hline Early terminated procedure & $1(0.5)$ & $1(0.5)$ & 0.999 \\
\hline
\end{tabular}


related bleeding regardless of its definition $[11,16,17$, 20-22, 26-28], but no major or serious bleeding events occurred with any patient in these reports. The bleeding rate observed in this study, $4.8 \%$, seems to be higher than those of prior studies, but we adopted a stricter criterion for reporting this complication (any bleeding requiring further intervention) than have other studies (bleeding necessitating premature termination of the procedure or a bleeding amount of $\geq 30 \mathrm{ml}$ ) [11, 26]. With regard to our study aim of comparing safety signals between the biopsy-first and brushing-first strategies, the complication profiles and rates for R-EBUSguided procedures were similar between the two groups of patients. Thus, the two procedure strategies studied herein were indistinguishable in terms of both efficacy and adverse events for patients with peripheral pulmonary malignancy.

In line with previous studies $[4,10,11,17,18]$, ours found that probe position is a major determinant of the diagnostic yield of R-EBUS-guided TBB for peripheral pulmonary malignancy. This finding reinforces the importance of navigating the probe to the desired position that is visualized within the target lesion. In this study, the R-EBUS probe was more likely to be placed within the malignancy in patients in the brushing-first group, so the difference between probe positions may complicate the comparison of the diagnostic efficacy of the two study groups before adjusting for this confounder. However, in both the multivariate model and the subgroup analysis after taking probe position into consideration, the diagnostic sensitivity for peripheral pulmonary malignancy remained similar between the two strategies. This suggests the robustness of our study findings.

A couple of caveats pertaining to this study should be mentioned. First, the study findings represent a singlecenter experience with R-EBUS-guided TBB for peripheral pulmonary malignancy carried out by a well-developed bronchoscopy team; thus, the results may not be generalized to other settings, such as a less-experienced institution or a lower-level healthcare facility. Nonetheless, as the first study to deal with this issue, we hope our report will encourage more large-scale and elaborate studies in this field. Second, our study was retrospective, and as such, the choice of procedure strategy was at the discretion of the in-charge pulmonary faculty. In this regard, a selection bias may exist; however, baseline features of the participants were nearly balanced between the biopsy-first and brushing-first groups, and further statistical adjustment did not identify any significant differences in the study outcomes between the two groups.

\section{Conclusions}

In summary, the timing of bronchial brushing, either before or after biopsy, for peripheral pulmonary malignancy, at least in experienced hands, did not influence the diagnostic yield of R-EBUS-guided TBB plus brushing. Moreover, a similarly favorable safety signal was observed between the two strategies. Therefore, current evidence indicates that in patients with peripheral pulmonary lesions suspected of being malignant, either biopsy-first or brushing-first would be a viable and acceptable strategy with respect to diagnostic sensitivity and safety during R-EBUSguided procedures.

\section{Abbreviations}

Cl: Confidence interval; CT: Computed tomography; OR: Odds ratio; REBUS: Radial endobronchial ultrasound; ROSE: Rapid on-site evaluation; TBB: Transbronchial biopsy

\section{Acknowledgements}

We thank the staff of the Eighth Core Lab, Department of Medical Research, National Taiwan University Hospital, for technical support during the study.

\section{Authors' contributions}

$\mathrm{CTH}$ and $\mathrm{CCH}$ designed the study, performed data curation and analysis, and drafted the manuscript. YJT analyzed and interpreted the data, and revised the manuscript critically for important intellectual content. CJY

conceptualized and designed the study, and critically revised the manuscript for important intellectual content. All authors read and approved the final manuscript.

Funding

Not applicable.

\section{Availability of data and materials}

The datasets used and/or analyzed during the current study are available from the corresponding author on reasonable request.

\section{Ethics approval and consent to participate}

The Research Ethics Committee of National Taiwan University Hospital approved the protocol and waived informed consent given the retrospective nature of the study and the lack of patient safety concerns.

Consent for publication

Not applicable.

\section{Competing interests}

The authors declare that they have no competing interests.

\section{Author details}

${ }^{1}$ Department of Internal Medicine, National Taiwan University Hospital, No. 7 Chung-Shan South Rd, Taipei 100, Taiwan. ${ }^{2}$ Graduate Institute of Clinical Medicine, College of Medicine, National Taiwan University, Taipei, Taiwan. ${ }^{3}$ Graduate Institute of Biomedical and Pharmaceutical Science, College of Medicine, Fu Jen Catholic University, New Taipei City, Taiwan.

Received: 11 June 2019 Accepted: 15 October 2019

Published online: 31 October 2019

References

1. Schuhmann M, Eberhardt R, Herth FJ. Endobronchial ultrasound for peripheral lesions: a review. Endosc Ultrasound. 2013;2:3-6.

2. Asano F, Eberhardt R, Herth FJ. Virtual bronchoscopic navigation for peripheral pulmonary lesions. Respiration. 2014;88:430-40.

3. Huang CT, Ruan SY, Liao WY, Kuo YW, Lin CY, Tsai YJ, Ho CC, Yu CJ. Risk factors of pneumothorax after endobronchial ultrasound-guided transbronchial biopsy for peripheral lung lesions. PLoS One. 2012;7:e49125.

4. Huang CT, Ruan SY, Tsai YJ, Ho CC, Yu CJ. Experience improves the performance of endobronchial ultrasound-guided transbronchial biopsy for peripheral pulmonary lesions: a learning curve at a medical centre. PLoS One. 2017;12:e0179719. 
5. Steinfort DP, Khor YH, Manser RL, Irving LB. Radial probe endobronchial ultrasound for the diagnosis of peripheral lung cancer: systematic review and meta-analysis. Eur Respir J. 2011;37:902-10.

6. Yoshikawa M, Sukoh N, Yamazaki K, Kanazawa K, Fukumoto S, Harada M, Kikuchi E, Munakata M, Nishimura M, Isobe H. Diagnostic value of endobronchial ultrasonography with a guide sheath for peripheral pulmonary lesions without X-ray fluoroscopy. Chest. 2007;131:1788-93.

7. Roth $\mathrm{K}$, Eagan TM, Andreassen AH, Leh F, Hardie JA. A randomised trial of endobronchial ultrasound guided sampling in peripheral lung lesions. Lung Cancer. 2011:74:219-25.

8. Hou G, Miao Y, Hu XJ, Wang W, Wang QY, Wu GP, Wang EH, Kang J. The optimal sequence for bronchial brushing and forceps biopsy in lung cancer diagnosis: a random control study. J Thorac Dis. 2016;8:520-6.

9. Lee HS, Kwon SY, Kim DK, Yoon HI, Lee SM, Lee JH, Lee CT, Chung HS, Han SK, Shim YS, et al. Bronchial washing yield before and after forceps biopsy in patients with endoscopically visible lung cancers. Respirology. 2007:12:277-82

10. Huang CT, Ho CC, Tsai YJ, Yu CJ, Yang PC. Factors influencing visibility and diagnostic yield of transbronchial biopsy using endobronchial ultrasound in peripheral pulmonary lesions. Respirology. 2009;14:859-64.

11. Kurimoto N, Miyazawa T, Okimasa S, Maeda A, Oiwa H, Miyazu Y, Murayama M. Endobronchial ultrasonography using a guide sheath increases the ability to diagnose peripheral pulmonary lesions endoscopically. Chest. 2004;126:959-65.

12. Huang CT, Tsai YJ, Liao WY, Wu PC, Ho CC, Yu CJ, Yang PC. Endobronchial ultrasound-guided transbronchial biopsy of peripheral pulmonary lesions: how many specimens are necessary? Respiration. 2012;84:128-34.

13. Huang CT, Tsai YJ, Ho CC, Yu CJ. Atypical cells in pathology of endobronchial ultrasound-guided transbronchial biopsy of peripheral pulmonary lesions: incidence and clinical significance. Surg Endosc. 2019;33:1783-8.

14. Huang CT, Tsai YJ, Ho CC, Yu CJ. The value of repeat radial-probe endobronchial ultrasound-guided transbronchial biopsy after initial nondiagnostic results in patients with peripheral pulmonary lesions. BMC Pulm Med. 2017;17:132.

15. Bilaceroglu S, Kumcuoglu Z, Alper H, Osma E, Cagirici U, Gunel O, Bayol U, Celikten E, Perim K, Kose T. CT bronchus sign-guided bronchoscopic multiple diagnostic procedures in carcinomatous solitary pulmonary nodules and masses. Respiration. 1998;65:49-55.

16. Ost DE, Ernst A, Lei X, Kovitz KL, Benzaquen S, Diaz-Mendoza J, Greenhill S, Toth J, Feller-Kopman D, Puchalski J, et al. Diagnostic Yield and Complications of Bronchoscopy for Peripheral Lung Lesions. Results of the AQulRE Registry. Am J Respir Crit Care Med. 2016;193:68-77.

17. Kikuchi E, Yamazaki K, Sukoh N, Kikuchi J, Asahina H, Imura M, Onodera Y, Kurimoto N, Kinoshita I, Nishimura M. Endobronchial ultrasonography with guide-sheath for peripheral pulmonary lesions. Eur Respir J. 2004;24:533-7.

18. Yamada N, Yamazaki K, Kurimoto N, Asahina H, Kikuchi E, Shinagawa N, Oizumi S, Nishimura M. Factors related to diagnostic yield of transbronchial biopsy using endobronchial ultrasonography with a guide sheath in small peripheral pulmonary lesions. Chest. 2007;132:603-8.

19. Fielding DI, Robinson PJ, Kurimoto N. Biopsy site selection for endobronchial ultrasound guide-sheath transbronchial biopsy of peripheral lung lesions. Intern Med J. 2008;38:77-84.

20. Herth FJ, Eberhardt R, Becker HD, Ernst A. Endobronchial ultrasound-guided transbronchial lung biopsy in fluoroscopically invisible solitary pulmonary nodules: a prospective trial. Chest. 2006;129:147-50.

21. Chung YH, Lie CH, Chao TY, Wang YH, Lin AS, Wang JL, Lin MC. Endobronchial ultrasonography with distance for peripheral pulmonary lesions. Respir Med. 2007;101:738-45.

22. Eberhardt R, Ernst A, Herth FJ. Ultrasound-guided transbronchial biopsy of solitary pulmonary nodules less than 20 mm. Eur Respir J. 2009;34:1284-7.

23. Chen $\mathrm{CH}$, Cheng WC, Wu BR, Chen CY, Chen WC, Hsia TC, Liao WC, Tu CY, Shih CM, Hsu WH, et al. Improved diagnostic yield of bronchoscopy in peripheral pulmonary lesions: combination of radial probe endobronchial ultrasound and rapid on-site evaluation. J Thorac Dis. 2015;7:S418-25.

24. Chen A, Chenna P, Loiselle A, Massoni J, Mayse M, Misselhorn D. Radial probe endobronchial ultrasound for peripheral pulmonary lesions. A 5-year institutional experience. Ann Am Thorac Soc. 2014;11:578-82.

25. Wang Memoli JS, Nietert PJ, Silvestri GA. Meta-analysis of guided bronchoscopy for the evaluation of the pulmonary nodule. Chest. 2012; 142:385-93.
26. Hayama M, Izumo T, Matsumoto Y, Chavez C, Tsuchida T, Sasada S. Complications with endobronchial ultrasound with a guide sheath for the diagnosis of peripheral pulmonary lesions. Respiration. 2015;90:129-35.

27. Herth FJ, Ernst A, Becker HD. Endobronchial ultrasound-guided transbronchial lung biopsy in solitary pulmonary nodules and peripheral lesions. Eur Respir J. 2002;20:972-4.

28. Paone G, Nicastri E, Lucantoni G, Dello lacono R, Battistoni P, D'Angeli AL, Galluccio G. Endobronchial ultrasound-driven biopsy in the diagnosis of peripheral lung lesions. Chest. 2005;128:3551-7.

\section{Publisher's Note}

Springer Nature remains neutral with regard to jurisdictional claims in published maps and institutional affiliations.
Ready to submit your research? Choose BMC and benefit from:

- fast, convenient online submission

- thorough peer review by experienced researchers in your field

- rapid publication on acceptance

- support for research data, including large and complex data types

- gold Open Access which fosters wider collaboration and increased citations

- maximum visibility for your research: over $100 \mathrm{M}$ website views per year

At BMC, research is always in progress.

Learn more biomedcentral.com/submissions 